\title{
Paternalismus und Citizenship
}

\author{
Herausforderungen professioneller Urteilsbildung im Sozialinvestionsstaat
}

Die große Bedeutung, die der Frage von professionellen Urteilen und Maßnahmeentscheidungen in der Sozialen Arbeit zugeschrieben wird, dürfte damit zusammenhängen, dass sie keine, auf trennscharfe diagnostische Semantiken angewiesene spezialisierte Leistung in einem ausdifferenzierten Funktionskontext ist.

$\mathrm{E}$ her erbringt Soziale Arbeit ,unspezifische Hilfen` (vgl. Bommes und Scherr 2000) mit Blick auf deutungs- und interpretationsbedürftige Praktiken und Situationen, die als morphologisch vielfältige und konstitutiv durch Sinngebungsprozesse imprägnierte Lebensführungsproblematiken in Erscheinung treten.

\section{Das Paternalismusproblem Sozialer Arbeit}

Unterstellt man vor diesem Hintergrund sozialwissenschaftlich naiv, dass es Fachkräften in der Sozialen Arbeit im Großen und Ganzen darum geht, ihren Adressat_innen zu helfen, besteht das professionelle Kardinalproblem bei (Inferenz-)Urteilen im technologisch nicht angemessen bearbeitbaren Problem des $\mathrm{Pa}$ ternalismus. Damit sind Eingriffe angesprochen, die Einschränkungen bestimmter Komponenten der Freiheit von Akteur_innen in deren eigenem Interesse bzw. im Sinne deren Woblergehens beinhalten.

\begin{tabular}{l} 
Holger Ziegler \\
$\begin{array}{l}\text { Universität Bielefeld, } \\
\text { Bielefeld, Deutschland } \\
\text { *1974; Dr., Professor für Soziale Arbeit an der Fakultät für } \\
\text { Erziehungswissenschaft der Universität Bielefeld. } \\
\text { holger.ziegler@uni-bielefeld.de }\end{array}$ \\
\hline
\end{tabular}

Zusammenfassung Der Beitrag argumentiert, dass das Paternalismusproblem ein Kernproblem professioneller Urteilsbildung als Grundlage einer angemessenen Praxis darstellt. Dieses Problem wird durch eine (normative) Ausrichtung des wohlfahrtsstaatlichen Arrangements gerahmt, das sich in Richtung eines sozialinvestionsstaatlichen Begründungsrahmens verschiebt. Dabei erfahren sozialpädagogische Wohlfahrtsleistungen eine veränderte Relevanz, die offenbar nicht dem vergleichsweise steuerungsresistenten professionellen Ermessen und Urteilen unterstellt werden soll.

Schlüsselwörter Autonomie, Begründung von Eingriffen, Citizenship, Paternalismus, Sozialinvestition, Würde
Auch wenn sich Soziale Arbeit bemühen mag, den Wünschen ihrer Adressat_innen zu entsprechen, ist im Kern davon auszugehen, dass helfende Eingriffe der Sozialen Arbeit paternalistisch sind. Auch dezidiert nutzer_innenorientierten Formulierungen Sozialer Arbeit, wie sie etwa Gertrud Oelerich und Andreas Schaarschuch vorlegen, geht es um die ko-produktive Erzeugung von Gebrauchswerten für die Subjekte und nicht um eine unkommentierte Erfüllung von (Kund_innen-) Wünschen. Selbst aus einer finanzwissenschaftlichen Perspektive, die keine Probleme hat, Nutzer_innen als Kund_innen oder Konsument_innen zu modellieren, ist die Sozialen Arbeit ein Paradebeispiel für die Produktion sog. meritorischer Güter, für die eine öffentliche Bereitstellung und Allokation - auch jenseits von Marktversagen - alleine deshalb als zweckdienlich gilt, weil seitens der Konsument_innen falsche oder unvorteilhafte Entscheidungen zu befürchten sind. Bei vielen Leistungen der Kinder- und Jugendhilfe will der Staat, dass die Leistungsadressat_innen die Leistungen konsumieren. Er wirkt aktiv und gegebenenfalls - z. B. mit dem Gesetz zur Erleichterung familiengerichtlicher Maßnahmen bei Gefährdung des Kindeswohls von 2008 - auch mit dem berühmten, steele of law' auf eine solche Konsumtion hin.

Wenn von einer Orientierung an den Wünschen der Betroffenen die Rede ist, geht es typischerweise eher darum, zwischen dem, was die Betroffenen wollen und dem was gut für sie ist zu vermitteln (entsprechend ist eher von ,Partizipation', ,Mitentscheidung' etc. als von Kund_innensouveränität die Rede). In manchen Fällen mag beides mehr oder weniger identisch sein. Dann macht es entsprechend wenig Unterschied, ob sich Soziale Arbeit an subjektiven Wünschen oder dem Wohlergehen bzw. den ,wohlverstandenen Interessen', dem Gebrauchswert etc. orientiert. Sofern aber davon auszugehen ist, dass der Rekurs auf,Wohlergehen' dann maßgeblich wäre, wenn das, was Adressat_innen wün- 


\section{Extrablick: Urteilsbildung in der Sozialen Arbeit}

schen und das was ,gut' für sie sein soll, nicht korrespondieren würde, kann man auch in einem solchen Fall eher von einem , latenten Paternalismus ${ }^{1}$ als von einer anti-paternalistische Perspektive sprechen.

Wenn es nun konstitutiv für paternalistische Eingriffe ist, dass ihre Ziele wohlwollend gegenüber den Adressat_innen sind, worin besteht dann das, Problem'? Paternalistische Eingriffe zur Förderung des Wohlergehens einer Person ziehen deren Willkürfreiheit in Mitleidenschaft und verletzen das (liberale) Gebot der Neutralität öffentlicher Institutionen gegenüber der Frage einer gelingenden Lebensführung von Bürger_innen. Für eine streng liberale Perspektive ist Soziale Arbeit schon alleine deswegen verdächtig, weil sie gegenüber der gelingenden Lebensführung nicht neutral ist, sondern verspricht diese zu befördern. Aber auch wenn man Soziale Arbeit nicht generell in Abrede stellt, bleibt Paternalismus erstens hinsichtlich der Behauptung zu wissen, was gut für Andere ist und zweitens mit Blick auf eine Übergehung von Selbstbestimmungsansprüchen problematisch. Das Paternalismusproblem stellt sich insofern vor allem normativ, aus einer im weitesten Sinne politisch liberalen Perspektive.

Bei der Rede von ,liberal‘ muss man weniger an ,NeoLiberalismus', sondern eher an das denken, was der Marxist Erik Olin Wright (2015, S. 67) ausführt: „Der Wert, der der Demokratie zugrunde liegt, ist der Wert der Selbstbestimmung, ist die Vorstellung, dass Menschen ibr Leben selbst kontrollieren, anstatt dass es durch andere kontrolliert wird. Dies schließt die individuelle Freiheit ein, Entscheidungen zu treffen, die ihr Leben als eigenständige Personen betreffen, und die Fähigkeit, an kollektiven Entscheidungen mitzuwirken, die das Leben als Mitglied einer umfassenderen Gemeinschaft beeinflussen. (... D)ie Ideen individueller Freiheit und Demokratie (teilen) grundsätzlich den gleichen Grundwert".

Wenn Selbstbestimmung demnach als ein zentraler Aspekt eines gelingenden Lebens gelten muss, stehen (möglicherweise) rechtfertigbare paternalistische Eingriffe in der Nachweispflicht, autonomiefunktional zu sein. Im Kern läuft das Argument darauf hinaus, dass sie die Würde der Betroffenen achten müssen, selbst über die Verwirklichung von Zuständen, Praktiken und Projekten zu verfügen, die sie vernünftigerweise wertschätzen. Im Begriff ,vernünftigerweise ‘ liegt der Hund begraben: Die Tendenz, missliebige Ansprüche und Perspektiven von Adressat_innen als ,unvernünftig' oder ,unrealistisch' abzutun und zu übergehen, ist ja in der Regel gerade das, was fachliche Paternalismuskritik zu Recht evoziert: Solche Paternalismusrechtfertigungen lassen praktisch jeden unerwünschten Eingriff zu, gehen Hand in Hand mit einem kognitiven Elitismus und kranken offensichtlich daran, dem Würdeanspruch nicht gerecht zu werden. Ironischerweise sind es gerade perfektionistische Theoretiker_innen, die darauf bestehen, dass Vernünftigkeit kein überbordend kognitivistisches und elitäres Vernunftkonzept verlangt, sondern ,lediglich“ voraussetzt, dass die ,adherents [... of a] conception of the good [...] are stably disposed to affirm it and it is the product of their efforts to find meaning or value in life" (Wall 2012). Nicht was Fachkräfte gut oder nützlich finden, sondern die - und nur die - Konzeption des Guten der Betroffenen selbst sei es, der rechtfertigbare paternalistische Eingriffe zu folgen haben, wenn sie zwischen dem artikulierten Wünschen und dem Wohl der Adressat_innen vermitteln.

Die Möglichkeit, Urteile in der Sozialen Arbeit auf die Konzeption des Guten ihrer Adressat_innen zu beziehen, setzt Fallverstehen und insbesondere die Rekonstruktion der Bedeutungen und Gewichtungen voraus, die Adressat_innen ihren je eigenen Lebensführungsprojekten zuweisen.

Am ehesten ist dies in einem professionalistischen Modus der Leistungserbringung zu erwarten. Damit ist nicht gesagt, dass professionelle Fachkräfte in ihren Entscheidungen und Fall- bzw. Bedürfnisinterpretationen den genannten Rechtfertigungsanforderung gerecht werden. Allerdings stellen standardisierte Maßnahmen, die vor der Intervention ihr Ziel bestimmen und dann prüfen, ob die Maßnahme ,wirksam' ist, die Adressat_innen von Zustand A nach Zustand B zu bewegen, einen nicht rechtfertigbaren Paternalismus auf Dauer. Folgt man Wright, verletzen sie dabei auch demokratische Zentralwerte. Man darf davon ausgehen, dass derzeit rekonstruierbare Entwicklungstendenzen in der Sozialen Arbeit auch den ,Citizenship'-Status ihrer Adressat_innen berühren.

\section{Sozialinvestition und Citizenship}

Diese Entwicklungen hängen mit einer Konzipierung von Wohlfahrt als produktive Sozialinvestition in $\mathrm{Hu}-$ mankapital - insbesondere von Kindern und Familien sowie mit der damit verknüpften Tendenz zusammen, sozial-strukturelle Problemlagen in einer personen- und verhaltensorientierten Weise zu problematisieren. Beides mündet zunächst in eine politisch dezidiert gewollte Bedeutungszunahme sozialer Dienste.

Wohlfahrtsökonomisch ist bereits aufgrund früher erworbener und langfristig bindender Berechtigungen gerade in sozialversicherungsförmig aufgebauten Wohlfahrtsstaaten eine kontinuierliche Steigerung von Ausgaben für monetäre Wohlfahrtsleistungen bei einem schrumpfenden Spielraum diskretionärer ${ }^{3}$, flexib- 
ler Ausgabenposten erwartbar. Tatsächlich ist aber laut OECD-Statistik der Anteil monetärer Wohlfahrtsleistungen am Bruttoinlandsprodukt in Deutschland seit 2000 deutlich geschrumpft, während der für (diskretionäre) Wohlfahrtsdienstleistungen stark gestiegen ist und für viele Bevölkerungsgruppen die Höhe von Transferzahlung eingeholt und z. T. auch übertroffen hat. Der Sozialdienstleistungssektor wies in den letzten 20 Jahren die stärkste Wachstumsdynamik aller Berufssektoren auf. Von 2006 auf 2019 hat sich alleine in der Kinder- und Jugendhilfe das Personal mehr als verdoppelt. Von 2000 bis 2019 sind die Ausgaben von ca. 18,5 auf knapp 55 Mrd. Euro gestiegen. Europaweit hat sich der enge statistische Zusammenhang von Ausgaben für monetäre und dienstleistungsförmige Wohlfahrtsleistungen entkoppelt und Deutschland gilt diesbezüglich als ein Beispiel für einen ,Trade-Off‘ zwischen sozialprotektiven und ,sozialinvestiven' Wohlfahrtsausgaben.

Diese Verschiebung mag auch mit einer veränderten Struktur sozialer Problemlagen zu tun haben. Allerdings zeichnen Metaanalysen nach, dass selbst für gemeinhin als sozialarbeitsrelevant geltende Problemlagen die Effektstärken monetärer Transferleistungen empirisch auf Augenhöhe mit sozio-edukativer Maßnahmen liegen (vgl. Cooper und Stewart 2013). Zumal anzunehmen ist, dass dienstleistungsbasierte Sozialinvestitionspolitiken insgesamt weniger, pro-poor ${ }^{\star}$ sind und ein "less redistributive profile than traditional transfers" aufweisen (Cantillon 2018, S. 21), spricht einiges für Jonathan Quongs (2011, S. 93) Einwand: „If the state favors a scheme where current economic injustices are rectified by state subsidies for valuable activities, rather than by a straightforward redistribution of wealth, that must be because the state does not believe the citizens to whom the redistribution is owed would spend their resources appropriately. In other words, the rationale for redistributing resources to the economically disadvantaged in services rather than in cash would be a paternalistic one". 4

Die Annahme, dass sich der Bedeutungsgewinn Sozialer Arbeit auch aus ,paternalistischen' Überlegungen speist, ist nicht unbegründet. Betont wird das Potenzial Sozialer Arbeit, auf Besonderheiten der persönlichen Situationen der Bürger_innen zu reagieren. Sie scheint geeignet um durch eine Verbesserung, Aufrechterhaltung oder Wiederherstellung von Kompetenzen, Verhaltensweisen, Einstellungen und anderen Dispositionen Chancen auf ,Teilhabe' an der Gesellschaft bzw. auf dem Arbeitsmarkt sowie die Produktivität in Arbeitsverhältnissen zu erhöhen und insgesamt die Anforderungen vorbeugender Sozialpolitiken - „präparieren statt reparieren“ und „vorbeugen statt heilen“
(Hemerijck 2017, S. 10) - zu erfüllen. Empirisch weisen Wohlfahrtsökonomen zumindest für fortgeschritten kapitalistische Gesellschaften einen substanziellen und positiven Zusammenhang von Ausgaben für Wohlfahrtsdienstleistungen und ökonomischem Wachstum aus, der sich für Absicherungsleistungen so nicht findet (vgl. Kim und Ahn 2020).

Allerdings wird auch geltend gemacht, dass eine sozialinvestive Wende nicht primär an der Form von Sozialleistungen, sondern vor allem deren Begründung und Ausrichtung berührt (vgl. Nolan 2013). Bezüglich der Gewährung sozialer Leistungen tritt die Einlösung des Versprechens eines Bürgerstatus - d. h. die Sicherung von Rechten und die Deckung von Ansprüchen und Bedarfen - gegenüber einer Re-Modellierung von Leistungen als Investitionen in den Hintergrund, die Erträge für wahlweise ,die Ökonomie' oder , die Gesellschaft ${ }^{\text {‘ }}$ generieren. Dabei sind inzwischen Versuche der Kommensuration der Effekte in Form kardinal vergleichbarer, monetärer Erträge verbreitet: Analysen sozialer Folgekosten in Staatskanzleien, bundesweite KostenNutzen-Analysen aller familienpolitscher Maßnahmen und schließlich bilanzanalytische Verfahren zur Berechnungen des Social Return on Investment (SROI) auf der Ebene von Einzelmaßnahmen. Dass die methodische Qualität solcher Analysen in einigen wenigen Fällen zwar beeindruckend, in der Mehrzahl aber schlechterdings lächerlich ist, ist weniger erheblich als der Umstand, dass offenbar Verfahren zur ex-ante-Kalkulation der Effizienz von Leistungsgewährungen im Sinne von Investitionsentscheidungen entwickelt werden, die an die Seite oder gar an die Stelle von Bezugsgrößen, wie z. B. den universellen Zugang zu Leistungen oder die Garantie politischer und sozialer Rechte, treten.

In einem als (,sozial'-)renditeorientierten Anbieter von Dienstleistungen verstandenen Wohlfahrtsstaat stellt sich die Frage professioneller Entscheidungskunst bestenfalls als Steuerungsproblem. Dabei geht es nicht darum, dass die Leistungen Sozialer Arbeit nicht anerkannt werden. Im Gegenteil: Sie gelten als ,wichtige Sozialinvestitionen - zu wichtig, als dass Sozialarbeiter_innen mit ihren amorphen und offenbar uneinheitlichen Professionskriterien und Mandatszuschreibungen darüber befinden sollen.

Protagonist_innen dieser Umgestaltung greifen zwar häufig das ,paternalismuskritische“ Argument auf, das Unterstützungs-, Kontroll- und ggf. auch Sanktionsentscheidungen oft von persönlichen Einstellungen und Ideologien der Fachkräfte abhängen, aber die Einschränkungen des diskretionären Spielraums von Professionellen durch bürokratisch-managerieller Kontroll- und Standardisierungsprozedere des New Public 


\section{Extrablick: Urteilsbildung in der Sozialen Arbeit}

Management sind realistischerweise weniger auf den Schutz von Gleichheit und Equity gerichtet, sondern darauf, dass die Maßnahmen S.M.A.R.T. in der politisch gewünschten Form und entsprechend der gesetzten Ziele - und eben nicht als kontigentes Produkt von Aushandlungen von Professionellen und Nutzer_innen - erbracht werden. Auch empirisch gibt es wenig Zweifel daran, dass Einschränkung von professionalistischen Entscheidungs- und Ermessenspielräumen weniger mit dem Schutz von Rechten oder der Diskriminierungsfreiheit der Betroffenen, sondern eher mit pejorativen Adressat_innenbildern und verstärkten Kontroll- und Sanktionsorientierungen einhergehen.

Vor diesem Hintergrund überrascht, dass die Sozialen Arbeit weiterhin geneigt ist, die eigene ,Systemrelevanz', die Notwendigkeit einer besseren Ausfinanzierung, die mangelnde Anerkennung und den Fachkräftemangel zu betonen. Das mag alles irgendwie auch stimmen, ist aber insofern ,geschenkt', wie all dies im Zuge einer Umgestaltung des Sozialstaats in einen dienstleistungsbasierten Sozialinvestitionsstaat ohnehin auf der Agenda steht. Diese Umgestaltung ist zwar eher im Gange als vollzogen, aber die entscheidende Frage der Sozialen Arbeit für die nächsten Dekaden dürfte weniger lauten, ob und inwiefern sie relevant ist, sondern in welcher Weise sie das zentrale, von ihr betraute demokratische Gut gestaltet: Social Citizenship.

Eingegangen. 7. Mai 2021

Angenommen. 11. Mai 2021

Funding. Open Access funding enabled and organized by Projekt DEAL.

Open Access. Dieser Artikel wird unter der Creative Commons Namensnennung 4.0 International Lizenz veröffentlicht, welche die Nutzung, Vervielfältigung, Bearbeitung, Verbreitung und Wiedergabe in jeglichem Medium und Format erlaubt, sofern Sie den/die ursprünglichen Autor(en) und die Quelle ordnungsgemäß nennen, einen Link zur Creative Commons Lizenz beifügen und angeben, ob Änderungen vorgenommen wurden.

Die in diesem Artikel enthaltenen Bilder und sonstiges Drittmaterial unterliegen ebenfalls der genannten Creative Commons Lizenz, sofern sich aus der Abbildungslegende nichts anderes ergibt. Sofern das betreffende Material nicht unter der genannten Creative Commons Lizenz steht und die betreffende Handlung nicht nach gesetzlichen Vorschriften erlaubt ist, ist für die oben aufgeführten Weiterverwendungen des Materials die Einwilligung des jeweiligen Rechteinhabers einzuholen.

Weitere Details zur Lizenz entnehmen Sie bitte der Lizenzinformation auf http://creativecommons.org/licenses/by/4.0/deed.de.

1. Wenn z. B. eine fiktive Jugendliche in der Heimerziehung bekunden würde, sie ziehe es vor, „¿Quién Mató a Sara?" auf Netflix zu schauen statt in die Schule zu gehen, ist die typische Reaktion auf
Seiten der Fachkräfte in der Regel nicht: „Machs dir schon mal auf dem Fernsehsessel bequem, ich bring dir gleich 'en Päckchen Chips dazu".

2. Die Vernunft einer Vorstellung des Guten setzt also voraus, dass deren Anhänger_innen geneigt sind, diese Vorstellung dauerhaft zu vertreten und sie das Produkt ihrer Bemühungen ist, Sinn oder Wert im Leben zu finden.

3. Diskretionäre Ausgaben sind - im Gegensatz zu sog. obligatorischen Ausgaben (zu diesen zählen insbesondere Transferzablungen an Individuen) - Ausgaben, die die durch politische Willensentscheidung veräußert oder vermieden werden können.

4. Wenn der Staat ein System bevorzugt, bei dem bestehende wirtschaftliche Ungerechtigkeiten durch die Förderung wertvoller Aktivitäten und nicht durch eine direkte Umverteilung des Reichtums behoben werden, dann liegt das daran, dass der Staat nicht glaubt, dass die Bürger_innen, denen die Umverteilung zugute kommt, ihre Ressourcen angemessen ausgeben würden. Mit anderen Worten, eine Umverteilung von Ressourcen an wirtschaftlich Benachteiligte in Form von Dienstleistungen statt Geld wäre paternalistisch begründet.

\section{Literatur}

Bommes, M., \& Scherr, A. (2000). Soziale Arbeit, sekundäre Ordnungsbildung und die Kommunikation unspezifischer Hilfsbedürftigkeit. In R. Merten (Hrsg.), Systemtheorie Sozialer Arbeit. Opladen: Leske + Budrich.

Cantillon, B. (2018). Social security and poverty reduction in rich welfare state. Antwerpen: Herman Deleeck Centre for Social Policy.

Cooper, K., \& Stewart, K. (2013). Does money affect children's outcomes? London: Joseph Rowntree Foundation.

Hemerijck, A. (2017). The uses of social investment. Oxford: Oxford University Press.

Kim, S. W., \& Ahn, S. H. (2020). Social investment effects of public education, health care, and welfare service expenditures on economic growth. Asian Social Work and Policy Review, 14, 24-44.

Nolan, B. (2013). What use is ,social investment'? In Journal of European Social Policy, 23, 459-468.

Quong, J. (2011). Liberalism without perfection. New York: Oxford University Press.

Wall, S. (2012). Perfectionism in Moral and Political Philosophy. https:// plato.stanford.edu/archives/win2012/entries/perfectionism-moral/ E. Zalta (Hrsg.), The Stanford Encyclopedia of Philosophy. Zugegriffen: 2. April 2021

Wright, E. O. (2015). Durch Realutopien den Kapitalismus transformieren. In M. Brie (Hrsg.), Mit Realutopien den Kapitalismus transformieren. Hamburg: VSA. 